\title{
Alcohol consumption and all-cause mortality in older adults in Spain: an analysis accounting for the main methodological issues
}

\author{
Rosario Ortolá 1,2 (D), Esther García-Esquinas ${ }^{1,2}$, Esther López-García ${ }^{1,2,3}$, Luz M. León-Muñoz ,2, \\ José R. Banegas ${ }^{1,2}$ \& Fernando Rodríguez-Artalejo ${ }^{1,2,3}$
}

Department of Preventive Medicine and Public Health, Universidad Autónoma de Madrid and Idipaz, Madrid, Spain,' CIBER of Epidemiology and Public Health (CIBERESP), Madrid, Spain ${ }^{2}$ and IMDEA Food Institute. CEI UAM+CSIC, Madrid, Spain ${ }^{3}$

\begin{abstract}
Background and aims Observational evidence that light-to-moderate alcohol consumption lowers mortality is questioned because of potential selection biases and residual confounding. We assess the association between alcohol intake and all-cause death in older adults after accounting for those methodological issues. Methods Data came from 3045 individuals representative of the non-institutionalized population aged $\geq 60$ years in Spain. Participants were recruited in 2008-10, when they reported current and life-time alcohol intake; drinkers were classified as occasional $(<1.43 \mathrm{~g} /$ day $)$, light $(\geq 1.43$ but $<20 \mathrm{~g} /$ day for men and $\geq 1.43$ but $<10 \mathrm{~g} /$ day for women), moderate $(\geq 20$ but $<40 \mathrm{~g} /$ day for men and $\geq 10$ but $<20 \mathrm{~g} /$ day for women) or heavy ( $\geq 40 \mathrm{~g} /$ day for men and $\geq 24 \mathrm{~g} /$ day for women)/binge. Participants were followed-up to 2017 to assess vital status. In analyses, ex-drinkers were removed from the abstainer group and were classified according to their life-time intake to address the 'abstainer bias'. Moreover, analyses were replicated in individuals without functional limitations, and excluded deaths in the first year of follow-up, to address reverse causation. Also, occasional drinkers were used as reference in some analyses to reduce the "healthy drinker/survivor' bias. Results were adjusted for many covariates to minimize residual confounding. Results Compared with never-drinkers, the hazard ratio (95\% confidence interval) of mortality for light drinkers was $1.05(0.71-1.56)$ and $1.20(0.72-2.02)$ in those without functional limitations. Corresponding values for moderate drinkers were 1.28 (0.81$2.02)$ and 1.55 (0.87-2.75) and for heavy/binge drinkers 1.85 (1.07-3.23) and 2.15 (1.09-4.22). Results were consistent when occasional drinkers were used as reference. Among drinkers without functional limitations, the hazard ratio $(95 \%$ confidence interval) of mortality per $10 \mathrm{~g} /$ day of alcohol was 1.12 (1.02-1.23). Conclusion After accounting for potential biases, light-to-moderate drinking among people 60+ years of age appears to have no statistically significant benefit on mortality compared with abstention from alcohol. By contrast, heavy/binge drinking shows a higher death risk compared with abstention from alcohol. Alcohol intake appears to have a positive dose-response with mortality among drinkers.
\end{abstract}

Keywords Alcohol, confounding, mortality, older adults, reverse causation, selection bias.

Correspondence to: Rosario Ortolá, and Fernando Rodríguez Artalejo, Department of Preventive Medicine and Public Health, School of Medicine, Universidad Autónoma de Madrid, Calle del Arzobispo Morcillo 4, 28029 Madrid, Spain. E-mail: ortolarosario@gmail.com; fernando.artalejo@uam.es Submitted 7 March 2018; initial review completed 23 April 2018; final version accepted 24 July 2018

\section{INTRODUCTION}

Alcohol consumption is a leading cause of health problems, ranking among the top five risk factors for disease, disability and death throughout the world [1,2]. Although in observational studies regular consumption of low-to-moderate amounts of alcohol has been associated with lower allcause mortality, due mainly to reduced cardiovascular deaths [3-5], the quality of the evidence rendered by these studies has been questioned due to potential selection biases and residual confounding [6-10].

One of these selection biases is the 'abstainer' bias, whereby the apparently lower mortality of moderate drinkers could be explained by the higher death risk of the abstainers, as they include ex-drinkers who quit alcohol because of poor health. One approach to reduce this bias is to remove ex-drinkers from the group of abstainers and consider them as a distinct category $[3,8,11]$, but this 
may create another bias from over-representation of healthier individuals among drinkers compared with non-drinkers [7]. Thus, another proposed solution to the 'abstainer' bias has been to re-classify ex-drinkers into other drinking categories according to their usual alcohol intake in the past [12].

Another type of selection bias is the 'healthy drinker/survivor' bias, which may underestimate the risk of death among drinkers compared with non-drinkers [11] because cohort studies, particularly of older adults, over-represent healthier drinkers who could also have survived the deleterious effects of alcohol. This bias could be palliated by using occasional drinkers as the reference group in the analyses [13-15], because it has been argued that, compared to non-drinkers, occasional drinkers may have life-style and health characteristics more similar to those observed in moderate drinkers while a biological effect of very low alcohol intake by occasional drinkers seems implausible [8,9,11]. Also, given that current drinking may reflect health status, this bias could also be palliated by re-classification of drinkers according to past consumption.

Also, it is possible that part of the association observed in cohort studies is due to health status influencing alcohol consumption, rather than the opposite [16]. This situation, known as reverse causation, can be addressed entirely only by experimental studies, but could be palliated by restricting the analyses to those in good health status (e.g. older adults without functional limitations) and excluding deaths occurring during the first years of followup. Finally, comparability of health behaviours and status between categories of drinkers and abstainers could be improved by adequate adjustment for potential confounders.

Recent research on the association between alcohol consumption and mortality addressing the above methodological issues has been conducted mainly in Anglo-Saxon countries [8-10,15,17-20]. However, the drinking patterns as well as the life-style and health characteristics of drinkers versus non-drinkers in those countries could differ from those in the Mediterranean basin, where moderate wine consumption during meals is traditionally considered part of a healthy life-style for all social classes [21].

Therefore, this work examined the association between alcohol consumption and the risk of all-cause death in community-dwelling older adults in Spain, while addressing some of the methodological issues believed to bias such a relationship. Specifically, in the analyses, ex-drinkers are removed from the abstainer group and re-classified according to life-time alcohol intake. Also, analyses exclude deaths during the first year of follow-up, are replicated in individuals without functional limitations and, in some cases, use occasional drinkers as reference. Lastly, analyses are adjusted for many covariates to reduce residual confounding.

\section{METHODS}

\section{Study design and population}

Data were taken from participants in the ENRICA study (Study on Nutrition and Cardiovascular Risk in Spain), a population-based investigation conducted between June 2008 and October 2010. Participants were selected by stratified cluster sampling of the community-dwelling adult population of Spain. Information was collected using a telephone interview to obtain data on socio-demographic factors, life-style and morbidity, and two home visits to collect biological samples, perform a physical examination and obtain a diet history $[21,22]$. The response rate among individuals aged $\geq 60$ was $60 \%$, which is comparable to that in other national health examination surveys in Europe [23]. A new wave of data collection was conducted in 2012. Study participants were followed to 2017 to ascertain vital status (median follow-up of 7.8 years).

Study participants provided written informed consent, and the Clinical Research Ethics Committees of 'La Paz' University Hospital in Madrid and Hospital Clinic in Barcelona approved the study.

\section{Study variables}

\section{Alcohol consumption}

At baseline, usual consumption of alcoholic beverages in the previous year was estimated with a validated diet history, developed from the one used in the EPIC cohort study (European Prospective Investigation into Cancer and Nutrition) in Spain $[21,24]$. This diet history collected information on 34 alcoholic beverages and used photographs to help quantify portion sizes. Alcohol content of each beverage was estimated using standard composition tables. In Spain, the estimated alcohol standard unit is $10 \mathrm{~g}$ of pure alcohol [25].

According to the average alcohol intake, study participants were classified as: (a) never-drinkers: average alcohol intake of $0 \mathrm{~g} /$ day; (b) ex-drinkers: average alcohol intake of $0 \mathrm{~g}$ /day who answered 'I used to drink alcohol, but I quit' when asked which statement best described their alcohol consumption; (c) occasional drinkers: average alcohol intake $>0 \mathrm{~g} /$ day but $<1.43 \mathrm{~g} /$ day (equivalent to $<1$ standard unit/week); (d) light drinkers: average alcohol intake $\geq 1.43 \mathrm{~g} /$ day but $<20 \mathrm{~g} /$ day for men and $<10 \mathrm{~g} /$ day for women, and also those with average intake of $0 \mathrm{~g} /$ day who answered 'I usually drink alcohol (at least once a week)' when asked which statement best described their alcohol consumption; (e) moderate drinkers: average alcohol intake $\geq 20$ but $<40 \mathrm{~g} /$ day for men and $\geq 10$ but $<24 \mathrm{~g}$ /day for women; and (f) heavy/binge drinkers: average alcohol intake $\geq 40 \mathrm{~g} /$ day for men and $\geq 24 \mathrm{~g} /$ day for women, and also those who reported binge drinking, 
defined as an intake of $\geq 80 \mathrm{~g}$ of alcohol in men or $\geq 60 \mathrm{~g}$ in women during any drinking session in the preceding 30 days [26]. Accordingly, the definitions of drinkers are based not only on average alcohol intake, but also imply the absence or presence of binge drinking.

Life-time alcohol consumption was collected by asking the participants about the amount and frequency of consumption of the main types of alcoholic beverages in each decade of their life. Based on this information, participants were re-categorized as never-drinkers, occasional drinkers, light drinkers, moderate drinkers or heavy/binge drinkers [21].

A preference for a specific type of alcoholic beverage (wine or other) was acknowledged when more than $80 \%$ of alcohol intake was derived from such drink [21].

Baseline information on alcohol consumption was updated with the data collected in 2012, and cumulative alcohol intake was calculated as an average of baseline and 2012 alcohol intake.

\section{Mortality}

The number and dates of deaths in the cohort from baseline (2008-10) to 30 June 2017 were ascertained using the Spanish National Death Index, a free and easily accessible database with information on all people who die in Spain [27]. The specificity of the probabilistic matching algorithm used by this tool is close to $100 \%$. To improve sensitivity, the vital status of each participant was established in 2012, 2015 and 2017.

\section{Potential confounders}

At baseline we collected data on variables that, according to the literature, might be related to both alcohol consumption and mortality. Socio-demographic and life-style characteristics included sex, age, educational level, tobacco smoking, usual time spent watching TV and in other sedentary behaviours (sitting at the computer, sitting while commuting, lying in the sun, listening to music or reading), leisure-time physical activity expressed as metabolic equivalent (MET)-hours/week and diet quality assessed with the Mediterranean Diet Adherence Screener (MEDAS), excluding the wine component [28].

Morbidity was ascertained by asking the study participants if they had been diagnosed previously with cardiovascular disease (myocardial infarction, stroke or heart failure), hypertension, diabetes, cancer, chronic respiratory disease (asthma or chronic bronchitis), osteomuscular disease (osteoarthritis or arthritis) or depression requiring treatment. Participants also reported the number of medications they were taking, which were checked against drug packages by study staff, and were asked to rate their health as excellent, very good, good, fair or poor by answering the question: 'In general, how would you rate your health?'.
Health was then classified as optimal (excellent, very good or good) or suboptimal (fair or poor). Functional limitations in instrumental activities of daily living (IADL) were assessed with the Lawton \& Brody Scale [29]. Participants with functional limitations had a score $\leq 7$ for women and $\leq 4$ for men. Lastly, weight and height were measured in standardized conditions [30] and used to calculate the body mass index (BMI) as the weight in $\mathrm{kg}$ divided by the square of height in metres.

\section{Statistical analysis}

From the initial sample of 3511 individuals, we excluded 210 without information on alcohol consumption, 243 with missing data on potential confounders and 13 who had died in the first year of follow-up. Thus, the final analytical sample included 3045 individuals.

The association between baseline average alcohol intake (or life-time average alcohol intake) and the risk of death during follow-up was summarized with hazard ratios (HRs) and their 95\% confidence intervals (CIs), obtained from Cox proportional hazards regression models, with age as time-scale and individual starting follow-up times (age at baseline) treated as staggered entries. Two Cox models were built: model 1 was adjusted for sex and educational level, while model 2 adjusted additionally for the remaining potential confounders mentioned above. Never drinkers were taken as reference group, but we conducted further analyses with occasional drinkers as reference to address the 'healthy drinker/survivor' bias. Also, we used as main exposure the baseline daily intake of alcohol standard units (as a continuous variable) as well as the cumulative (average of baseline and 2012) alcohol intake; in this case, follow-up of participants started on the date of the 2012 assessment. Analyses were conducted on several groups of participants: (a) the total study sample; (b) a subset with no IADL limitations or (c) the sample of drinkers; and (d) a subset of drinkers with no IADL limitations.

We assessed whether the study results varied with sex, age $(<75, \geq 75)$ and self-reported health (optimal, suboptimal) by testing the statistical significance of interaction terms defined as the product of these variables by categories of alcohol drinking patterns, and we found no interactions. Thus, results are presented without disaggregation by those variables.

Statistical significance was set at two-sided $P<0.05$. Analyses were performed with Stata ${ }^{\circledR}$, version 13.1 .

\section{RESULTS}

A total of 327 of 3045 participants (10.7\%) died during the 7.8-year follow-up. Table 1 shows the main sociodemographic, life-style and clinical characteristics of study participants at baseline according to their vital status at 
Table 1 Socio-demographic, life-style and clinical characteristics of study participants at baseline, by vital status after 7.8 years.

\begin{tabular}{|c|c|c|c|c|c|c|}
\hline & \multicolumn{2}{|c|}{ Total $(n=3045)$} & \multicolumn{2}{|c|}{ Dead $(n=327)$} & \multicolumn{2}{|c|}{ Alive $(n=2718)$} \\
\hline & $n$ & $\%$ & $n$ & $\%$ & $n$ & $\%$ \\
\hline Men & 1395 & 45.1 & 205 & 61.7 & 1528 & 42.8 \\
\hline Age $\geq 75$ years & 628 & 21.4 & 165 & 51.2 & 463 & 17.3 \\
\hline \multicolumn{7}{|l|}{ Educational level } \\
\hline$\leq$ Primary & 1759 & 59.9 & 204 & 63.8 & 1555 & 59.3 \\
\hline Secondary & 711 & 22.9 & 70 & 20.3 & 641 & 23.3 \\
\hline University & 575 & 17.2 & 53 & 15.9 & 522 & 17.4 \\
\hline \multicolumn{7}{|l|}{ Tobacco smoking } \\
\hline Current smoker & 355 & 11.7 & 52 & 15.0 & 303 & 11.2 \\
\hline Former smoker & 903 & 29.2 & 130 & 40.6 & 773 & 27.7 \\
\hline Never smoker & 1787 & 59.1 & 145 & 44.4 & 1642 & 61.1 \\
\hline \multicolumn{7}{|c|}{ Leisure-time physical activity (MET-hours/week) } \\
\hline Lower tertile & 1217 & 40.5 & 167 & 49.3 & 1050 & 39.3 \\
\hline Intermediate tertile & 821 & 26.5 & 75 & 24.3 & 746 & 26.7 \\
\hline Upper tertile & 1007 & 33.0 & 85 & 26.4 & 922 & 34.0 \\
\hline \multicolumn{7}{|l|}{ Time watching TV (hours/day) } \\
\hline Lower tertile & 1547 & 50.0 & 139 & 41.7 & 1408 & 51.1 \\
\hline Intermediate tertile & 698 & 22.7 & 69 & 20.5 & 629 & 23.0 \\
\hline Upper tertile & 800 & 27.3 & 119 & 37.8 & 681 & 25.9 \\
\hline \multicolumn{7}{|c|}{ Other sedentary behaviours ${ }^{\mathrm{a}}$ (hours/day) } \\
\hline Lower tertile & 1025 & 34.1 & 134 & 39.9 & 891 & 33.3 \\
\hline Intermediate tertile & 1067 & 34.3 & 109 & 35.2 & 958 & 34.2 \\
\hline Upper tertile & 953 & 31.6 & 84 & 24.9 & 869 & 32.5 \\
\hline \multicolumn{7}{|l|}{ MEDAS (excluding wine) } \\
\hline Lower tertile & 1237 & 41.2 & 159 & 49.1 & 1078 & 40.2 \\
\hline Intermediate tertile & 1281 & 42.3 & 121 & 38.2 & 1160 & 42.8 \\
\hline Upper tertile & 527 & 16.5 & 47 & 12.7 & 480 & 17.0 \\
\hline Cardiovascular disease $^{\mathrm{b}}$ & 173 & 5.9 & 39 & 11.1 & 134 & 5.2 \\
\hline Hypertension & 1988 & 66.8 & 232 & 70.5 & 1756 & 66.3 \\
\hline Diabetes & 525 & 17.9 & 93 & 25.0 & 432 & 17.0 \\
\hline Cancer & 68 & 2.4 & 14 & 4.7 & 54 & 2.1 \\
\hline Respiratory disease $^{c}$ & 233 & 8.3 & 37 & 12.2 & 196 & 7.8 \\
\hline Osteomuscular disease $^{\mathrm{d}}$ & 1532 & 50.5 & 171 & 52.8 & 1361 & 50.1 \\
\hline Depression requiring treatment & 272 & 8.8 & 20 & 5.5 & 252 & 9.3 \\
\hline Number of medications $>3$ & 1023 & 35.7 & 164 & 52.4 & 859 & 33.4 \\
\hline \multicolumn{7}{|l|}{ Body mass index $\left(\mathrm{kg} / \mathrm{m}^{2}\right)$} \\
\hline$<25$ & 596 & 19.6 & 74 & 24.0 & 522 & 19.0 \\
\hline $25-<30$ & 1446 & 45.8 & 153 & 43.9 & 1293 & 46.0 \\
\hline$\geq 30$ & 1003 & 34.6 & 100 & 32.1 & 903 & 35.0 \\
\hline Optimal self-rated health ${ }^{\mathrm{e}}$ & 1906 & 60.2 & 157 & 46.7 & 1749 & 62.0 \\
\hline Functional limitations ${ }^{\mathrm{f}}$ & 380 & 11.9 & 107 & 29.9 & 273 & 9.4 \\
\hline
\end{tabular}

${ }^{a}$ Sitting at the computer, sitting while commuting, lying in the sun, listening to music or reading. ${ }^{b}$ ischaemic heart disease, stroke or heart failure. ${ }^{c}$ asthma or

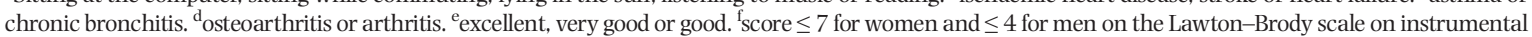
activities of daily living. MEDAS = Mediterranean Diet Adherence Screener; MET = metabolic equivalent.

the end of follow-up. Compared with participants who were alive at follow-up, those who died were more frequently male, older and current or former smokers, conducted less physical activity, spent more time watching TV but less time in other sedentary behaviours and had a lower diet quality. They also reported more morbidity and medication use, worse self-reported health and more disability. Supporting information, Tables S1-2 show the characteristics of participants according to alcohol intake.
The association between alcohol intake and risk of death is shown in Table 2 (and Supporting information, Tables S3-4 for full results on adjustment variables). Compared with never-drinkers, ex-drinkers showed a higher risk of death, both in the total sample (HR $=1.49 ; 95 \%$ $\mathrm{CI}=1.03-2.14)$ and in the subset of participants with no functional limitations ( $\mathrm{HR}=1.68$; 95\% CI $=1.08-2.60$ ). No statistically significant association was found between any of the drinker groups and the risk of death compared 


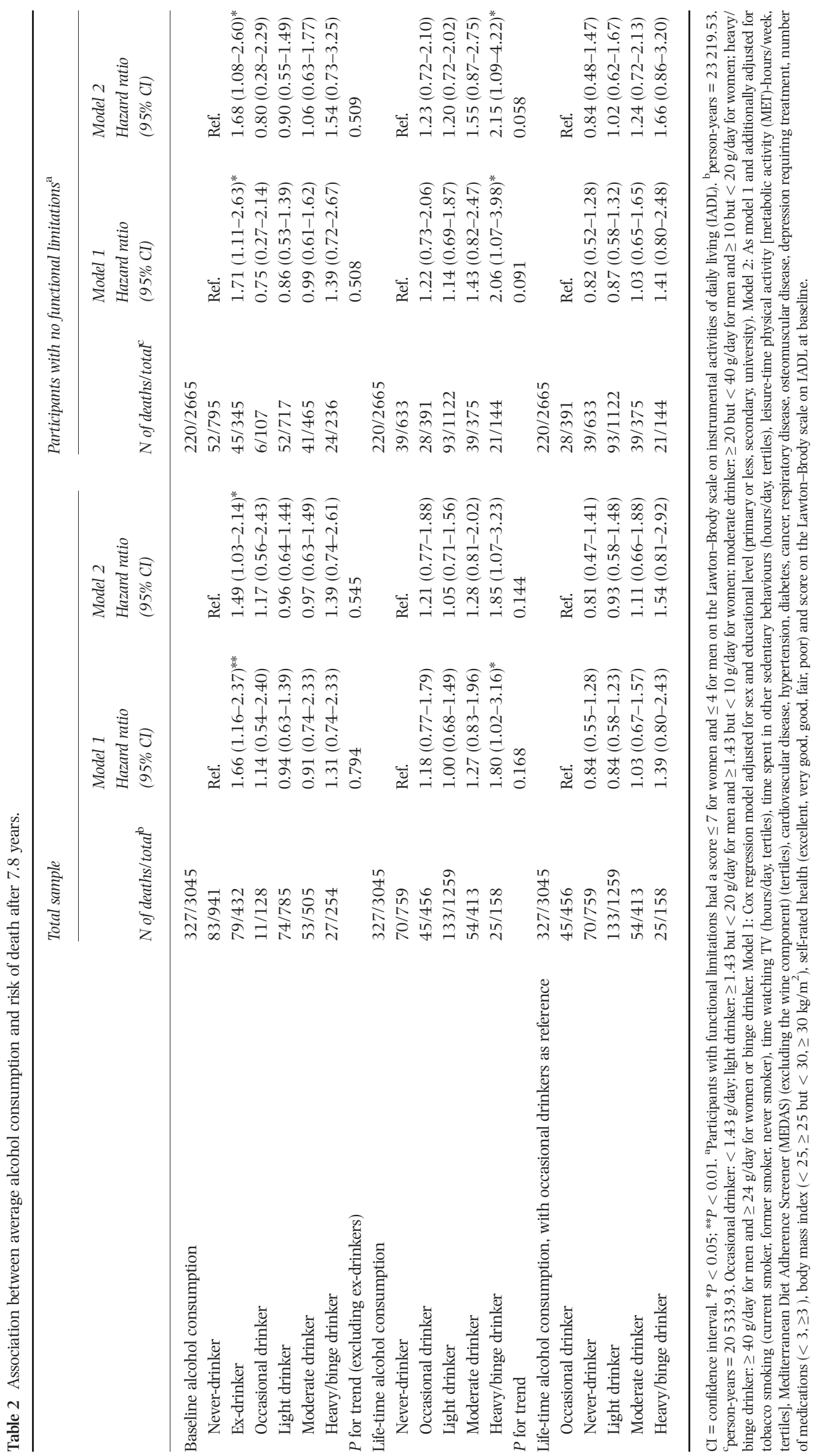


with never-drinkers, either in the total study sample or in those participants without IADL limitations.

When study participants were re-categorized according to life-time alcohol intake, heavy/binge drinkers showed higher mortality than never-drinkers, both in the total study sample $(\mathrm{HR}=1.85 ; 95 \% \mathrm{CI}=1.07-3.23)$ and in the subset without IADL limitations (HR $=2.15 ; 95 \%$ $\mathrm{CI}=1.09-4.22$ ). Again, no statistically significant association was found for occasional, light or moderate drinkers, but weak evidence of an association with higher death risk was observed for moderate drinkers. When using occasional drinkers as reference, results were consistent overall (Table 2).

Finally, when analyses were restricted to drinkers, an increase in death risk per alcohol standard unit/day was found in those without IADL limitation, both for baseline alcohol intake $(\mathrm{HR}=1.10 ; 95 \% \mathrm{CI}=1.01-1.20)$ and for cumulative alcohol intake $(\mathrm{HR}=1.12$; $95 \% \mathrm{CI}=1.02-$ 1.23) (Table 3). Given that wine consumption has been associated specifically with lower mortality in some investigations [31-33], we also examined this issue without finding any relationship; among drinkers, model 2 HR (95\% CI) for wine versus other beverage preference was 1.20 (0.75-1.93) in the total sample, and $1.05(0.62-1.75)$ in those without IADL limitations.

\section{DISCUSSION}

In this study on a representative sample of communitydwelling older adults in Spain, no evidence was found that light/moderate drinking lowers all-cause mortality. By contrast, heavy/binge drinking was associated with higher death risk, and weak evidence of an association between moderate drinking and increased death risk was found.
Also, among drinkers, a positive dose-response relationship was identified for alcohol intake.

Other studies have brought into question the protective effect of low-to moderate alcohol consumption, including a recent analysis of almost 600000 current drinkers from 83 prospective studies [34], which found a curvilinear positive association between alcohol consumption and all-cause mortality, with a threshold for lowest risk of approximately $100 \mathrm{~g} /$ week. Interestingly, the association with cardiovascular disease was J-shaped and the association with myocardial infarction was inverse. Also, a Mendelian randomization study found that individuals with a genetic predisposition to drink less alcohol (carriers of ADH1B rs1229984 A-allele) had lower levels of risk factors for cardiovascular disease, as well as a lower risk for cardiovascular disease, independently of the amount of alcohol consumed [35]. This could contribute to the beneficial effect of alcohol on cardiovascular and all-cause mortality found in previous research.

Much debate has taken place concerning the best reference group to study the association between alcohol intake and mortality. Although it has been argued that occasional drinkers may represent a more appropriate reference group $[8,9,11]$, we did not conduct our main analyses using occasional drinkers as reference because of the small size of this group. Conversely, never-drinkers were used as reference and ex-drinkers were considered as a separate group and not in the abstainer group. In this analysis, no associations were found for occasional, light, moderate or heavy/binge drinkers, and ex-drinkers showed a higher risk of death than never-drinkers, which reflects the poorer health usually found in this group, whether or not related to alcohol consumption [4].

Nevertheless, considering ex-drinkers as a distinct category removes ex-drinkers from the abstainer group but also

Table 3 Association between the average amount of alcohol consumed by drinkers and risk of death.

\begin{tabular}{|c|c|c|c|c|c|c|}
\hline & \multicolumn{3}{|c|}{ Total sample of drinkers } & \multicolumn{3}{|c|}{ Drinkers with no functional limitations ${ }^{\mathrm{a}}$} \\
\hline & $\begin{array}{l}\text { N of deaths/ } \\
\text { Total }\end{array}$ & $\begin{array}{l}\text { Model } 1 \\
\text { Hazard ratio } \\
(95 \% \text { CI })\end{array}$ & $\begin{array}{l}\text { Model } 2 \\
\text { Hazard ratio } \\
(95 \% \text { CI })\end{array}$ & $\begin{array}{l}\text { N of deaths/ } \\
\text { Total }\end{array}$ & $\begin{array}{l}\text { Model } 1 \\
\text { Hazard ratio } \\
(95 \% \text { CI })\end{array}$ & $\begin{array}{l}\text { Model } 2 \\
\text { Hazard ratio } \\
(95 \% \text { CI })\end{array}$ \\
\hline $\begin{array}{l}\text { Baseline alcohol intake } \\
\text { (standard units }^{\mathrm{b}} / \text { day) }\end{array}$ & $165 / 1672$ & $1.03(0.95-1.12)$ & $1.06(0.97-1.16)$ & $123 / 1525$ & $1.07(0.99-1.16)$ & $1.10(1.01-1.20)^{*}$ \\
\hline 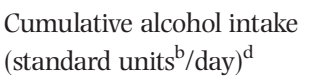 & $165 / 1672$ & $1.04(0.95-1.15)$ & $1.06(0.96-1.17)$ & $123 / 1525$ & $1.09(1.00-1.20)$ & $1.12(1.02-1.23)^{*}$ \\
\hline
\end{tabular}

$\mathrm{CI}=$ confidence interval; ${ }^{*} \mathrm{P}<0.05$. ${ }^{\mathrm{a}}$ Participants with functional limitations had a score $\leq 7$ for women and $\leq 4$ for men on the Lawton-Brody scale on instrumental activities of daily living (IADL). ${ }^{b}$ the estimated alcohol standard unit is $10 \mathrm{~g}$ of pure alcohol. ${ }^{c}$ median follow-up of 7.8 years. ${ }^{\mathrm{d}}$ follow-up of participants with average of baseline and 2012 alcohol intake started on the date of the 2012 data collection (median follow-up of 4.7 years).Model 1: Cox regression model adjusted for sex and educational level (primary or less, secondary, university). Model 2: As model 1 and additionally adjusted for tobacco smoking (current smoker, former smoker, never smoker), time watching TV (hours/day, tertiles), time spent in other sedentary behaviours (hours/day, tertiles), leisure-time physical activity (MET-hours/week, tertiles), Mediterranean Diet Adherence Screener (MEDAS) (excluding the wine component) (tertiles), cardiovascular disease, hypertension, diabetes, cancer, respiratory disease, osteomuscular disease, depression requiring treatment, number of medications $(<3, \geq 3)$, body mass index $\left(<25, \geq 25\right.$ but $\left.<30, \geq 30 \mathrm{~kg} / \mathrm{m}^{2}\right)$, self-rated health (excellent, very good, good, fair, poor) and score on the Lawton-Brody scale on IADL at baseline. 
from the drinker groups, and given that people usually stop drinking due to poor health, this procedure leads to an over-representation of healthier individuals among drinkers compared with non-drinkers [11]. To overcome this issue, we used information on life-time alcohol consumption and re-categorized participants according to their previous level of alcohol intake. Interestingly, a higher risk of death was observed in heavy/binge drinkers compared with never-drinkers. This result highlights the relevance of data on life-time alcohol consumption. It is possible that life-time consumption may have been subjected to more error than current use, as each recall period was 10 years long and past intake could have been influenced by current intake. However, life-time alcohol consumption provides important information, as it would allow the examination of the effects of life-time cumulative exposure to alcohol [5].

This higher risk of death observed in heavy/binge drinkers is consistent with the increased risk of death per alcohol standard unit/day found in drinkers without IADL limitations. It has been argued that removing individuals with ill health from the analyses would bias the associations towards more favourable results for drinkers compared with non-drinkers, because the 'healthy drinker' bias is magnified [11]. In our analyses we found the opposite: risk estimates tended to be higher in the subset with no functional limitations than in the total sample, for all the categories of drinkers compared with never-drinkers. Therefore, removing participants with poor health status does not seem to have introduced additional bias; on the contrary, it has allowed some associations to become apparent, because it has probably mitigated reverse causation.

No associations were found when occasional drinkers were used as reference, not even for ex-drinkers. It has been suggested that occasional drinkers are a more appropriate reference group than life-time abstainers $[8,15]$, because they appear to have life-style and health characteristics more comparable with those of regular drinkers [9], while a poor health status has been reported for never-drinkers [36]. However, baseline characteristics of occasional drinkers in our study were more like those of neverdrinkers or even ex-drinkers than those of moderate drinkers (Supporting information, Tables S1-2), suggesting that many of them may be individuals who have reduced their alcohol intake for health reasons.

Regarding other patterns of alcohol consumption, no association between beverage preference and all-cause mortality was found in our study. Our results are in line with a meta-analysis that did not found a greater benefit of wine versus other beverages on the main cardiovascular risk factors or on the incidence of cardiovascular disease [4,37], but are opposed to the results of a study in young university alumni in Spain that found a beneficial effect on mortality of wine preference that was independent of other aspects of the alcohol drinking pattern [31]. Other studies have also found a higher protective effect of wine on mortality than beer or spirits [32,33], so whether or not wine preference has a beneficial health effect is an unresolved issue.

\section{Strengths and limitations}

Among the strengths of this study is that participants were representative of the non-institutionalized older adult population of a whole country and that they underwent a long-term follow-up. Moreover, the causes of death observed in our cohort were very similar to those of the older population of Spain during the study period; the three main causes of death in our cohort were cardiovascular disease (30.6\% of all deaths), cancer $(27.1 \%)$ and respiratory disease $(16.6 \%)$, and the corresponding percentages for the Spanish population aged $\geq 60$ years were 32.0, 25.8 and $12.2 \%$. Also, alcohol consumption was obtained by using a validated diet history, with a Pearson correlation coefficient between the diet history and seven 24-hour recalls during 1 year for alcohol consumption of 0.65 [27]; moreover, life-time alcohol consumption was also reported, providing an estimate of life-time cumulative exposure to alcohol.

The main limitation of this study is that it was not designed to examine the effect of alcohol consumption on mortality, so it is not powered to detect small size effects, such as those of light-to-moderate alcohol consumption on all-cause mortality found in previous studies. The small size and the few death events observed in the group of occasional drinkers may also have reduced the reliability of results that used these drinkers as reference. The traditional drinking pattern of older adults in Spain consists of consuming wine during meals [24]; in fact, $68 \%$ study participants showed wine preference and $62 \%$ drank only with meals. This pattern tends to define two separate populations, regular drinkers and non-drinkers, making occasional drinking relatively exceptional. Perhaps occasional drinkers could be a more appropriate reference group in other cultural and geographic contexts, where drinking with meals is not the norm in older adults. Because depression has been identified as a risk factor for heavy drinking [38], we adjusted our analyses for depression requiring treatment; however, our results may have underestimated the actual relationship, as alcohol consumption has also been shown to be causally related to depression $[39,40]$. Also, despite the known interactions between alcohol consumption and tobacco smoking with the risk for cancer [41] or between alcohol consumption and socio-economic status with all-cause mortality [42], we failed to find any of these interactions in our sample, due presumably to limited statistical power. Therefore, it is possible that our findings 
may not apply to higher-risk subgroups, such as heavy smokers or socio-economically disadvantaged populations. Even though we adjusted our analyses for smoking status, not accounting for the level of smoking may have led to residual confounding. An additional limitation is that lifetime alcohol consumption may not capture the transient effects of alcohol, specifically the acute effects of alcohol on injurious deaths as well as the reduction of cancer risk associated with declining alcohol intake over time. Also, we cannot entirely rule out reverse causation, despite the long follow-up period and the measures taken to reduce it, such as excluding participants who died in the first year or conducting analyses on participants with no functional limitations.

\section{CONCLUSIONS}

After accounting for potential biases (and confounders), this study did not provide evidence for the beneficial effect on all-cause mortality attributed previously to light-tomoderate alcohol consumption. It is possible that this issue could not be resolved definitively until the publication of the results from the Moderate Alcohol and Cardiovascular Health (MCH15) trial, which are expected by 2026 $[43,44]$. Until then, our results have important practical implications, because they suggest that even wellfunctioning older adults cannot obtain any mortality benefit from moderate alcohol intake. Because of this, as well as the high prevalence of chronic conditions aggravated by alcohol and the frequent use of alcohol-interacting drug treatments, it is sensible that older adults, who still have potentially many years to live in acceptable health, are not advised to drink alcohol to improve their health.

Conversely, heavy/binge drinking showed a detrimental impact on mortality. Consistent with this finding, a doseresponse relationship was identified for average alcohol intake, with an increased risk of death per alcohol standard unit/day in drinkers with no functional limitations.

\section{Declaration of interests}

This work was supported mainly by grant no. 02/2014 from the Plan Nacional sobre Drogas (Ministry of Health of Spain). Additional funding was obtained from FIS grants 12/1166 and 16/609 (Instituto de Salud Carlos III, State Secretary of R+D+I and FEDER/FSE), CIBERESP (grant 01/2016), the ATHLOS project (EU H2020Project ID: 635316) and the Salamander Project (JPI-A Healthy Diet for a Healthy Life, State Secretary of $\mathrm{R}+\mathrm{D}$ $+\mathrm{I}$ PCIN-2016-145). The funding agencies had no role in study design, data analysis, interpretation of results, manuscript preparation or in the decision to submit this manuscript for publication. We declare no other interests.

\section{References}

1. World Health Organization (WHO). The Global Status Report on Alcohol and Health 2014. Geneva: WHO; 2011 Available at: http://apps.who.int/iris/bitstream/10665/112736/1/97892 40692763_eng.pdf (accessed 12 January 2018) (Archived at http://www.webcitation.org/71doLO3wM on 13 August 2018).

2. Gakidou E., Afshin A., Abajobir A. A., Abate K. H., Abbafati C., Abbas K. M. et al. GBD 2016 Risk Factors Collaborators. Global, regional, and national comparative risk assessment of 84 behavioural, environmental and occupational, and metabolic risks or clusters of risks, 1990-2016: a systematic analysis for the Global Burden of Disease Study 2016. Lancet 2017; 390: 1345-422.

3. Di Castelnuovo A., Costanzo S., Bagnardi V., Donati M. B., Iacoviello L., de Gaetano G. Alcohol dosing and total mortality in men and women: an updated meta-analysis of 34 prospective studies. Arch Intern Med 2006; 166: 2437-45.

4. Ronksley P. E., Brien S. E., Turner B. J., Mukamal K. J., Ghali W. A. Association of alcohol consumption with selected cardiovascular disease outcomes: a systematic review and meta-analysis. BMJ 2011; 342: d671.

5. Jayasekara H., English D. R., Room R., MacInnis R. J. Alcohol consumption over time and risk of death: a systematic review and meta-analysis. Am J Epidemiol 2014; 179: 1049-59.

6. Chikritzhs T., Stockwell T., Naimi T., Andreasson S., Dangardt F., Liang W. Has the leaning tower of presumed health benefits from 'moderate' alcohol use finally collapsed? Addiction 2015; 110: $726-7$.

7. Stockwell T., Chikritzhs T. Commentary: Another serious challenge to the hypothesis that moderate drinking is good for health? Int J Epidemiol 2013; 42: 1792-4.

8. Stockwell T., Zhao J., Panwar S., Roemer A., Naimi T., Chikritzhs T. Do 'moderate' drinkers have reduced mortality risk? A systematic review and meta-analysis of alcohol consumption and all-cause mortality. J Stud Alcohol Drugs 2016; 77: 185-98.

9. Knott C. S., Coombs N., Stamatakis E., Biddulph J. P. All cause mortality and the case for age specific alcohol consumption guidelines: pooled analyses of up to 10 population based cohorts. BMJ 2015; 350: h384.

10. Bergmann M. M., Rehm J., Klipstein-Grobusch K., Boeing H., Schütze M., Drogan D. et al. The association of pattern of lifetime alcohol use and cause of death in the European Prospective Investigation into Cancer and Nutrition (EPIC) study. Int J Epidemiol 2013; 42: 1772-90.

11. Naimi T. S., Stockwell T., Zhao J., Xuan Z., Dangardt F., Saitz R. et al. Selection biases in observational studies affect associations between 'moderate' alcohol consumption and mortality. Addiction 2017; 112: 207-14.

12. Liang W., Chikritzhs T. The association between alcohol exposure and self-reported health status: the effect of separating former and current drinkers. PLoS ONE 2013; 8: e55881.

13. Zeisser C., Stockwell T. R., ChikritzhsT. Methodological biases in estimating the relationship between alcohol consumption and breast cancer: the role of drinker misclassification errors in meta-analytic results. Alcohol Clin Exp Res 2014; 38: 2297-6.

14. Fillmore K. M., Kerr W. C., Stockwell T., Chikritzhs T., Bostrom A. Moderate alcohol use and reduced mortality risk: systematic error in prospective studies. Addict Res Theory 2006; 14: 101-32.

15. Park J. E., Ryu Y., Cho S. I. The effect of reference group classification and change in alcohol consumption on the 
association between alcohol consumption and cardiovascular disease. Alcohol Clin Exp Res 2017; 41: 379-87.

16. Holdsworth C., Mendonça M., Pikhart H., Frisher M., de Oliveira C., Shelton N. Is regular drinking in later life an indicator of good health? Evidence from the English Longitudinal Study of Ageing. J Epidemiol Community Health 2016; 70: 764-70.

17. Jayasekara H., MacInnis R. J., Hodge A. M., Hopper J. L., Giles G. G., Room R. et al. Alcohol consumption for different periods in life, intake pattern over time and all-cause mortality. J Public Health $(O x f)$ 2015; 37: 625-33.

18. Goulden R. Moderate alcohol consumption is not associated with reduced all-cause mortality. Am J Med 2016; 129 180-6.

19. Xi B., Veeranki S. P., Zhao M., Ma C., Yan Y., Mi J. Relationship of alcohol consumption to all-cause, cardiovascular, and cancer-related mortality in U.S. adults. J Am Coll Cardiol 2017; 70: 913-22.

20. Zhao J., Stockwell T., Roemer A., Naimi T., Chikritzhs T. Alcohol consumption and mortality from coronary heart disease: an updated meta-analysis of cohort studies. J Stud Alcohol Drugs 2017; 78: 375-86.

21. León-Muñoz L. M., Galán I., Donado-Campos J., SánchezAlonso F., López-García E., Valencia-Martín J. L. et al. Patterns of alcohol consumption in the older population of Spain, 2008-2010. J Acad Nutr Diet 2015; 115: 213-24.

22. Rodríguez-Artalejo F., Graciani A., Guallar-Castillón P., León-Muñoz L. M., Zuluaga M. C., Lopez-García E. et al. Rationale and methods of the Study on Nutrition and Cardiovascular Risk in Spain (ENRICA). Rev Esp Cardiol 2011; 64: 876-82.

23. Aromaa A., Koponen P., Tafforeau J., Vermeire C., HIS/HES Core Group Evaluation of health interview surveys and health examination surveys in the European Union. Eur J Public Health 2003; 13: 67-72.

24. Guallar-Castillón P., Sagardui-Villamor J., Balboa-Castillo T., Sala-Vila A., Ariza Astolfi M. J., Sarrión Pelous M. D. et al. Validity and reproducibility of a Spanish dietary history. PLoS ONE 2014; 9: e86074.

25. Rodríguez-Martos Dauer A., Gual Solé A., Llopis Llácer J. J. The 'standard drink unit' as a simplified record of alcoholic drink consumption and its measurement in Spain. Med Clin (Barc) 1999; 112: 446-50.

26. Soler-Vila H., Galán I., Valencia-Martín J. L., León-Muñoz L. M., Guallar-Castillón P., Rodríguez-Artalejo F. Binge drinking in Spain, 2008-2010. Alcohol Clin Exp Res 2014; 38: 810-9.

27. Ministerio de Sanidad, Servicios Sociales e Igualdad. National Death Index. Available at: https://www.msssi.gob.es/ en/estadEstudios/estadisticas/estadisticas/estMinisterio/IND_ TipoDifusion.htm (accessed 15 February 2018).

28. Schröder H., Fitó M., Estruch R., Martínez-González M. A., Corella D., Salas-Salvadó J. et al. A short screener is valid for assessing Mediterranean diet adherence among older Spanish men and women. J Nutr 2011; 141: 1140-5.

29. Lawton M. P., Brody E. M. Assessment of older people: selfmaintaining and instrumental activities of daily living. Gerontologist 1969; 9: 179-86.

30. Gutiérrez-Fisac J. L., Guallar-Castillón P., León-Muñoz L. M., Graciani A., Banegas J. R., Rodríguez-Artalejo F. Prevalence of general and abdominal obesity in the adult population of Spain, 2008-2010: the ENRICA study. Obes Rev 2012; 13: 388-92.

31. Gea A., Bes-Rastrollo M., Toledo E., Garcia-Lopez M., Beunza J. J., Estruch R. et al. Mediterranean alcohol-drinking pattern and mortality in the SUN (Seguimiento Universidad de Navarra) Project: a prospective cohort study. Br J Nutr 2014; 111: 1871-80.

32. Grønbaek M., Becker U., Johansen D., Gottschau A., Schnohr P., Hein H. O. et al. Type of alcohol consumed and mortality from all causes, coronaryheart disease, and cancer. Ann Intern Med 2000; 133: 411-9.

33. Tverdal A., Magnus P., Selmer R., Thelle D. Consumption of alcohol and cardiovascular disease mortality: a 16 year follow-up of 115,592 Norwegian men and women aged 4044 years. Eur J Epidemiol 2017; 32: 775-83.

34. Wood A. M., Kaptoge S., Butterworth A. S., Willeit P., Warnakula S., Bolton T. et al. Risk thresholds for alcohol consumption: combined analysis of individual-participant data for 599912 current drinkers in 83 prospective studies. Lancet 2018; 391: 1513-23.

35. Holmes M. V., Dale C. E., Zuccolo L., Silverwood R. J., Guo Y., Ye Z. et al. Association between alcohol and cardiovascular disease: Mendelian randomisation analysis based on individual participant data. BMJ 2014; 349: g4164.

36. Ng Fat L., Shelton N. Associations between self-reported illness and non-drinking in young adults. Addiction 2012; 107: 1612-20.

37. Brien S. E., Ronksley P. E., Turner B. J., Mukamal K. J., Ghali W. A. Effect of alcohol consumption on biological markers associated with risk of coronary heart disease: systematic review and meta-analysis of interventional studies. BMJ 2011; 342: d636.

38. Sacco P., Bucholz K. K., Harrington D. Gender differences in stressful life events, social support, perceived stress, and alcohol use among older adults: results from a national survey. Subst Use Misuse 2014; 49: 456-65.

39. Gea A., Beunza J. J., Estruch R., Sánchez-Villegas A., SalasSalvado J., Buil-Cosiales P. et al. Alcohol intake, wine consumption and the development of depression: the PREDIMED study. BMC Med 2013; 11: 192.

40. Bellos S., Skapinakis P., Rai D., Zitko P., Araya R., Lewis G. et al. Longitudinal association between different levels of alcohol consumption and a new onset of depression and generalized anxiety disorder: results from an international study in primary care. Psychiatry Res 2016; 243: 30-4.

41. McKee S. A., Weinberger A. H. How can we use our knowledge of alcohol-tobacco interactions to reduce alcohol use? Апnи Rev Clin Psychol 2013; 9: 649-74.

42. Katikireddi S. V., Whitley E., Lewsey J., Gray L., Leyland A. H. Socioeconomic status as an effect modifier of alcohol consumption and harm: analysis of linked cohort data. Lancet Public Health 2017; 2: e267-e276.

43. Mukamal K. J., Clowry C. M., Murray M. M., Hendriks H. F., Rimm E. B., Sink K. M. et al. Moderate alcohol consumption and chronic disease: the case for a long-term trial. Alcohol Clin Exp Res 2016; 40: 2283-91.

44. Moderate Alcohol and Cardiovascular Health (MCH15) trial. Availabe at: https://clinicaltrials.gov/ct2/show/ NCT03169530 (acccessed 28 February 2018).

\section{Supporting Information}

Additional supporting information may be found online in the Supporting Information section at the end of the article. 
Table S1 Socio-demographic, life-style and clinical characteristics of study participants at baseline, by baseline alcohol intake.

Table S2 Socio-demographic, life-style and clinical characteristics of study participants at baseline, by life-time alcohol intake.
Table S3 Association between baseline alcohol consumption and risk of death after 7.8 years.

Table S4 Association between life-time alcohol consumption and risk of death after 7.8 years. 